\title{
Photoelectron Backscattering from Silicon Anodes of Hybrid Photodetector Tubes
}

\author{
C. D'Ambrosio and H. Leutz
}

\begin{abstract}
The impact of photoelectron backscattering on spectral distributions measured with Hybrid Photodetector Tubes has been calculated. The calculations are based on the backscattering coefficient $\mu$, the average number of photoelectrons $\bar{N}_{\text {phel }}$ emitted from the photocathode, and on the distribution of the fractional photoelectron energy $q$ absorbed in silicon during the backscattering process. We obtained the following results: the average number of absorbed (measured) photoelectrons $\bar{N}_{\text {meas }}$ in the silicon anode amounts to $\sim \mathbf{8 8 \%}$ of the incident $\bar{N}_{\text {phel }}$. Photoelectron- and gamma-absorption peaks are broadened by a factor 1.043 due to backscattering. As an example, for Photo Multiplier Tubes, this broadening can amount to an average factor of 1.18 due to statistic and gain fluctuations on the dynode chain.
\end{abstract}

Index Terms-Backscattering, hybrid photodetector tubes.

\section{INTRODUCTION}

$\mathbf{H}$ YBRID Photodetector Tubes (HPT) [1] are equipped with photocathodes for light detection and their emitted photoelectrons are accelerated by potential differences $U$ of some $5-25 \mathrm{kV}$ toward a silicon anode. They strike the anode, which transfers the absorbed energy into electronic signals by generating on the average one electron-hole pair for each $\varepsilon=3.6 \mathrm{eV}$ of absorbed energy. The silicon anodes consist of one or few PIN diodes in Hybrid Photomultiplier Tubes (HPMT, Fig. 1) [2], of up to 1000 diodes in Multi Anode Photodetector (MAP)-tubes [3], and of finely segmented pixels in Imaging Silicon Pixel Array (ISPA)-tubes [4]. The surface of their single diodes ranges from a few square millimeters to $\sim 0.02 \mathrm{~mm}^{2}$ for the ISPA-tube. All HPT-anodes have in common that the photoelectrons impinging upon them are partly backscattered.

Electron backscattering has been measured by several authors ([5]-[9]) by observing single electrons at a time and describing their individual behavior. However, we will follow a different concept by treating a (Poissonian) collection of photoelectrons, released from the photocathode of an HPT by an incident light pulse. This process in HPT's was first pointed out and described in [2], in connection with photon counting measurements of light yield in scintillating fibers. More recently, a few papers have been published, which characterize the properties of scintillation crystal-HPT systems in $X$ - and $\gamma$-rays spectroscopy and imaging (see for example [10]-[12] and cited references therein). Moreover, high-energy physics experiments ${ }^{1}$ are adopting HPT's as photon detectors and often depend on

Manuscript received February 15, 2000; revised May 24, 2000.

The authors are with Cern, CH-1211, Geneva 23, Switzerland.

Publisher Item Identifier S 0018-9499(00)06549-7.

${ }^{1}$ To cite only a few, Compact Muon Solenoid and LHC-b experiments at CERN, Geneva, Switzerland, or the FINUDA experiment in LNF, Frascati, Italy. their photoelectron yield and intrinsic energy resolution. In this framework, the understanding of the role of backscatter processes in HPT's becomes crucial.

After an introduction to the processes involved in photon counting measurements with an HPT, we will describe in an analytical way the main properties of HPMT's spectra, when backscattering processes are accounted for. In particular, we will show how they affect the measured absorbed energy and the energy resolutions of their photoelectron- or total absorption peaks. Throughout this paper, we will use the fractional energy parameter $q$ and the energy proportional quantities $\bar{N}_{\text {phel }}$, which represent the average number of photoelectrons generated at the photocathode, and $\bar{N}_{\text {meas }}$, which is the effective average energy absorbed by the anode.

\section{THE BACKSCATTERING PROCESS}

Assuming that the backscattered photoelectrons move against an electric field between photocathode and anode that is approximately perpendicular to the HPT-anode surface, they follow ballistic trajectories. They reach their maximum vertical distance from the anode surface

$$
y_{\max }=d \sin ^{2} \delta
$$

with $\delta$ meaning the backscatter angle with respect to the anode plane and $d$ the distance between anode and photocathode. The maximum horizontal distance from the point of anode incidence amounts to

$$
x_{\max }=2 d \sin 2 \delta .
$$

At $45^{\circ}$ backscattering angle we obtain $y_{\max }=0.5 d$ and $x_{\max }=$ $2 d$.

A backscattered electron, returning to the anode plane, misses the anode itself, if $x<x_{\max }$ exceeds the anode dimension. This is mostly the case for HPMT's, which are operated in the cross-focusing mode (Fig. 1) and have silicon anodes of only a 2-mm diameter. These backscattered electrons contribute to the anode signal only with the energy loss they suffer inside the anode during the backscattering process. In the recorded spectra, this appears in the continuum present between resolved photoelectron peaks [2], [10]-[12].

The backscatter probability of an electron incident on a thick sample is expressed by the backscattering coefficient $\mu$. It is a function of the electron incidence angle $\vartheta$, the atomic number $Z$ of the sample, and the incident electron energy $E_{0}$. Within the photoelectron energy range of interest (10-25 keV) $\mu$ is fairly constant [6], [7]. For a uniformly illuminated HPMT, the angles $\vartheta$ of electron incidence range between 0 and $35^{\circ}$ (see Fig. 1) 


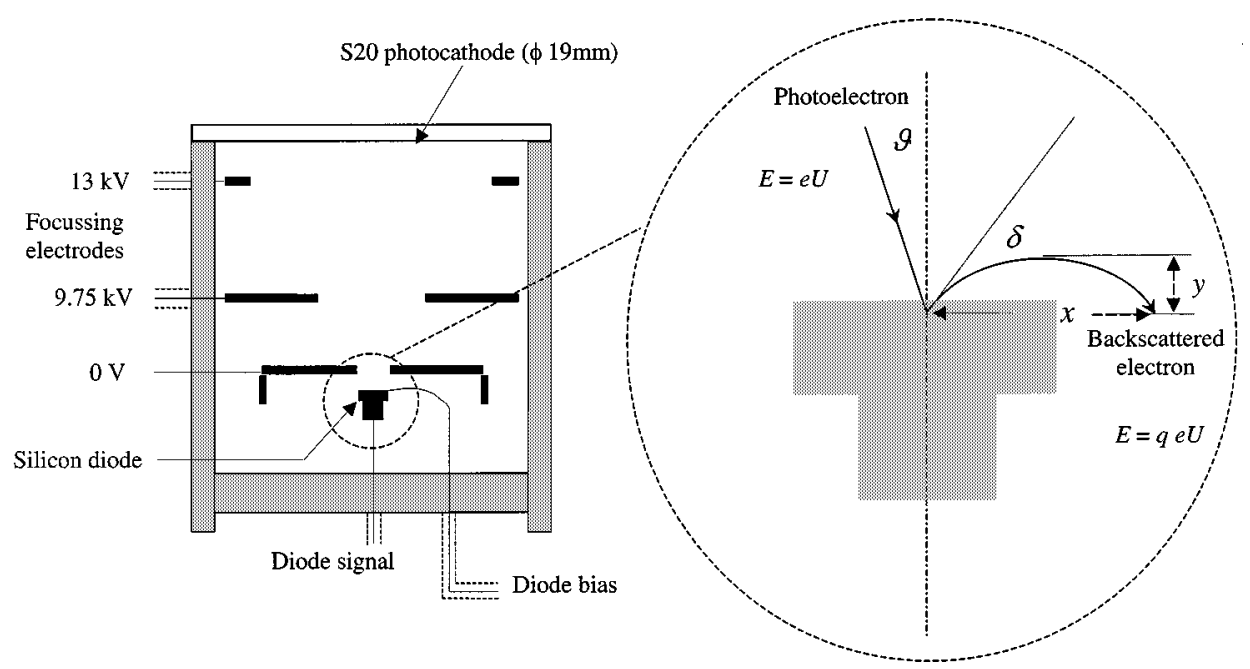

Fig. 1. Scheme of a cross-focused HPMT. In the enlarged area, the main quantities characterizing the backscattered electron are indicated.

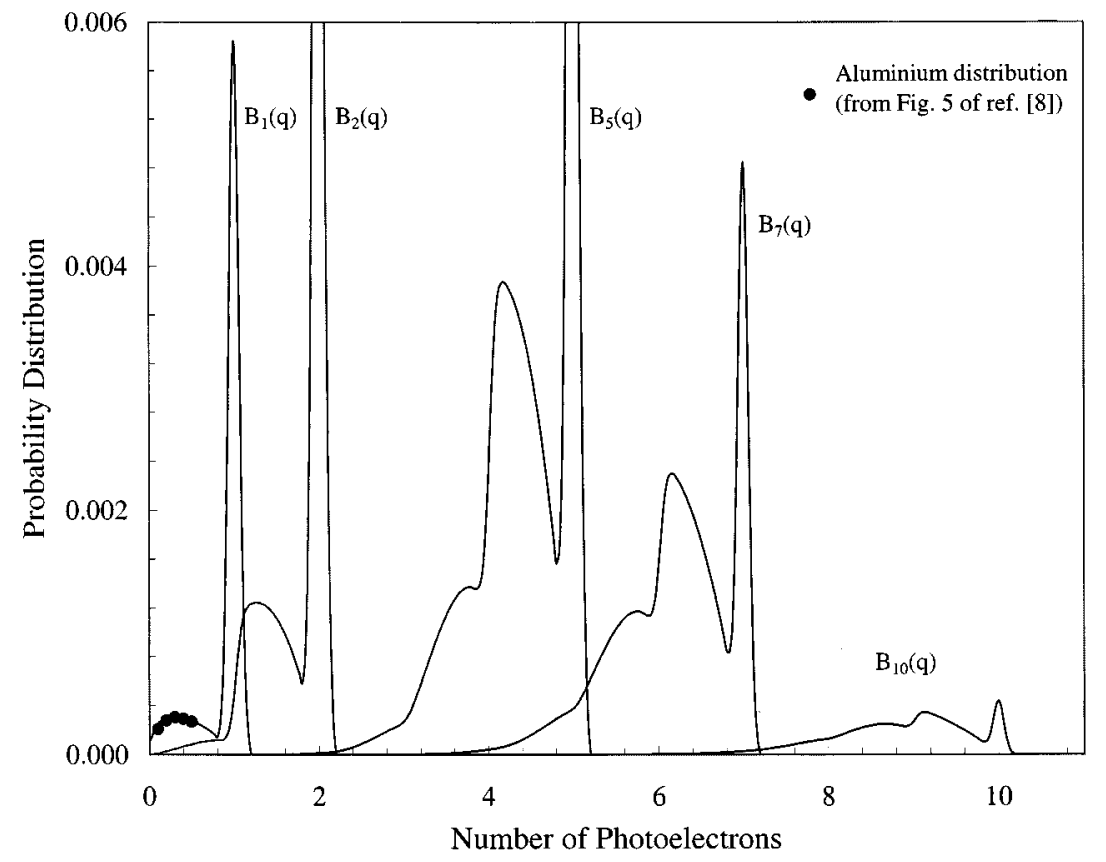

Fig. 2. Backscattering distributions $B_{m}(q)$ for $m=1,2,5,7$, and 10. For comparison, five measured points taken from [8, Fig. 5] are indicated. Electronic

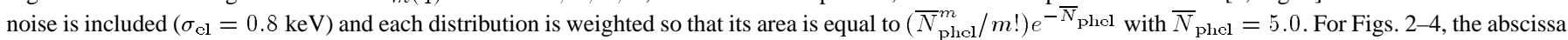
represents: - the continuous quantity $q$, which spans between 0 and $q_{m}$ for each $B_{m}(q)$, and - the integral quantity $m=q_{m}$ and ultimately the effective charge as measured by the electronics in calibrated photoelectron numbers.

with a weighted average angle of $\bar{\vartheta}=24^{\circ}$. From reference $[6$, Fig. 2] we obtain $\mu=0.20$ for $\bar{\vartheta}$. We will apply this value in the following calculations.

During the backscattering process, the electron can transfer to the substrate a factor $0 \leq q \leq 1$ of its incident energy $E_{0}$, leaving the sample with a backscatter energy $E_{b}=(1-q) E_{0}$. The probability for an electron to deposit a fraction between $q$ and $q+d q$ of its initial energy to the sample is $B_{1}(q)$ and was measured experimentally in [8] for various electron incident energies $E_{0}$ and materials.

For electron energies ranging between 10 and $15 \mathrm{keV}$ and according to [8, Fig. 5], the measured average fraction $\bar{E}_{b} / E_{0}$ of electrons, with incident energy $E_{0}$ and backscattered with an average energy $\bar{E}_{b}$, amounts to 0.65 when integrated over its backscatter angles, which agrees with the Monte Carlo simulation of Funsten et al. [9, (plotted in their Fig. 5)]. The fractional average energy loss of a backscattered photoelectron inside the silicon anode is therefore

$$
1-\frac{\bar{E}_{b}}{E_{0}}=\bar{q}=0.35 .
$$

This value is generally larger than the value $q_{\max }$ indicating the most probable value of the backscattering energy distribution. From the energy distribution of backscattered electrons shown in [8, Fig. 5] for aluminum $(Z=13)$, we obtain $q_{\max }=0.3$ which we apply for silicon $(Z=14)$. Finally, the average loss due to backscattering can roughly be estimated to $\sim \mu \bar{E}_{b} / E_{0}$ 


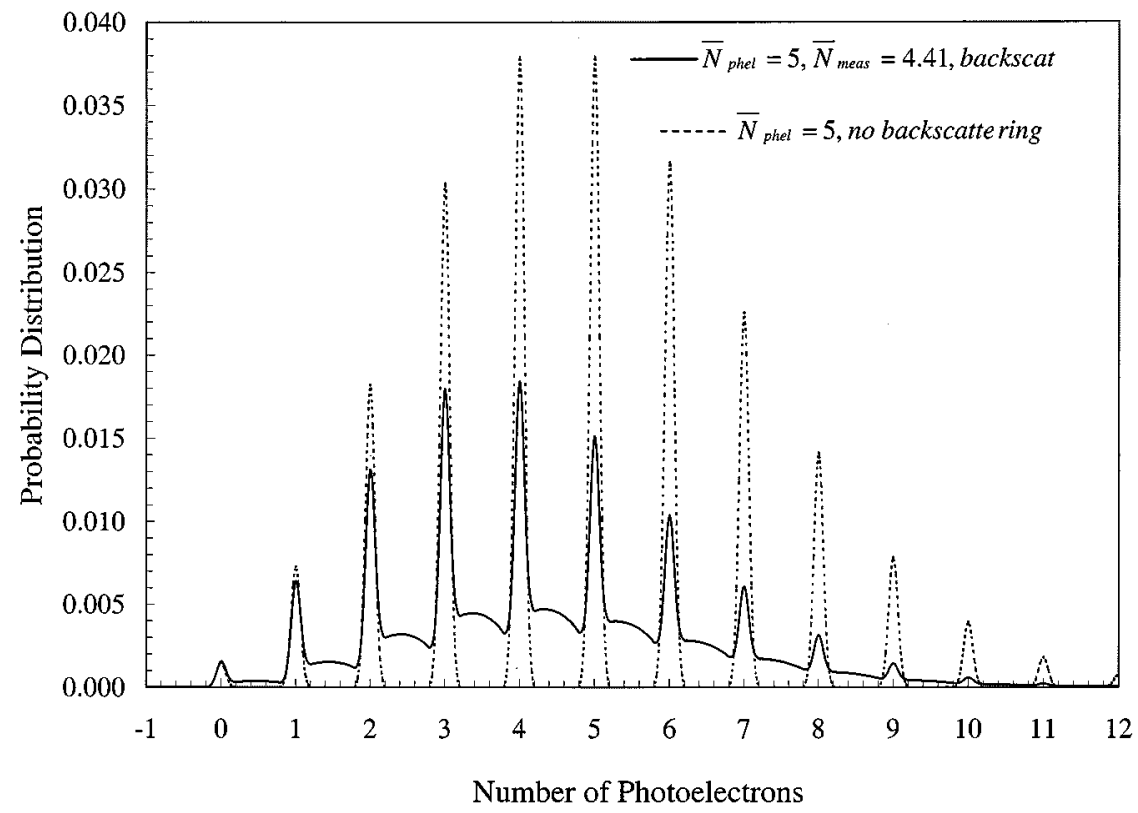

Fig. 3 Calculated spectra without and with backscattering. The electronic noise is convoluted $\left(\sigma_{\mathrm{el}}=0.8 \mathrm{keV}\right)$ for both distributions, $q_{\mathrm{max}}=0.30$ and $\mu=0.20$. The spectrum including backscattering is formed by the sum of all $B$ 's [see (I.1)], some of which are shown in Fig. 2.

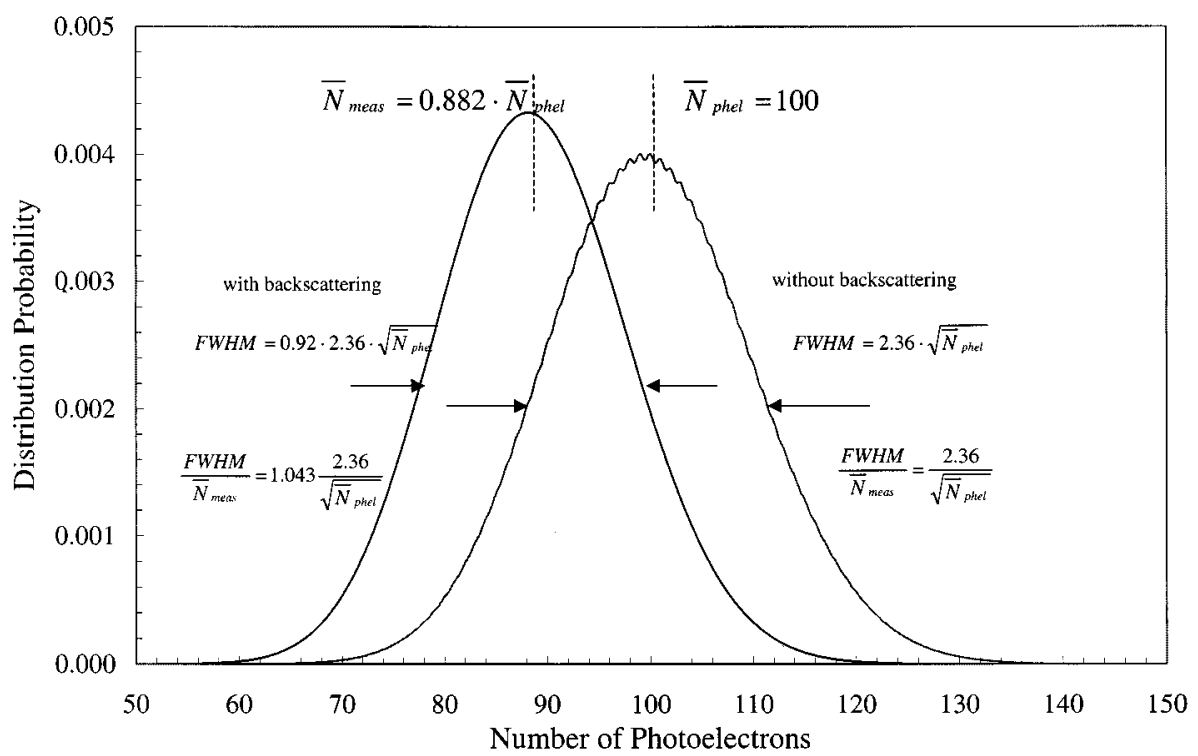

Fig. 4. The calculated spectra for $\bar{N}_{\text {phel }}=100$ with and without backscattering are shown. A slight indication of photoelectron peaks on top of the no-backscattering spectrum is still visible, in spite of the high average number of photoelectrons and of the intrinsic noise $\left[\sigma_{\text {int }} \sim 2.7 \mathrm{keV}\right.$, see $\left.(6 \mathrm{~b})\right]$. Indicated are all the important statistical quantities.

and amounts to 0.13 , which agrees again with the simulation shown in [9, Fig. 6].

In this paper, we will show how the measured pulse height distribution, characterized by the equivalent number of detected photoelectrons $\bar{N}_{\text {meas }}$, from an initial (Poissonian) distribution of electrons with energies $E_{0}$ incident on silicon, is affected by backscattering. We will start with a general distribution $B_{1}(q)$ for the backscattered electrons and then we will give an example of a backscattering distribution (Fig. 4 and Appendix III, where the distribution simulates that measured by [8, Fig. 5]). In particular, we will obtain a general relation between the average number of electrons released by the photocathode $\bar{N}_{\text {phel }}$ and the average number of electrons $\bar{N}_{\text {meas }}$ as measured by the silicon anode of the HPMT and a similar relation for the energy resolutions of the resulting spectra. Our goal is to give more physics insight to the meaning of the measured spectra and to obtain general relations, which can be easily extended to other types of HPT's. ${ }^{2}$

\section{How BACKSCATtering AfFeCtS THE ENERGY RESOLUTION}

For an ideal detector, light pulses with constant intensity, incident on an HPT-photocathode, produce an average number

\footnotetext{
${ }^{2}$ There is no attempt to achieve the best procedure to fit measured spectra, see for example [13].
} 
$\bar{N}_{\text {phel }}$ of photoelectrons. For $\bar{N}_{\text {phel }}$ small, we obtain with an HPMT a Poisson distribution with $m$ separated photoelectron peaks [2]. Each of these peaks is described by

$$
P_{m}\left(m, \bar{N}_{\text {phel }}\right)=\frac{\bar{N}_{\text {phel }}^{m}}{m !} e^{-\bar{N}_{\text {plel }}}
$$

Its envelope, summing for all the $m$ 's, represents the spectrum and its standard deviation amounts to

$$
\sigma=\sqrt{\bar{N}_{\text {phel }}}
$$

As $\bar{N}_{\text {phel }}$ becomes large, the photoelectron peaks seem to fuse more and more to a symmetric continuum whose contour approaches a Gaussian with standard deviation $\sigma=\sqrt{\bar{N}_{\text {phel }}}$ and continuous variable $x$

$$
P\left(x, \bar{N}_{\text {phel }}\right)=\frac{1}{\sqrt{2 \pi \sigma^{2}}} e^{-\left(\left(x-\bar{N}_{\text {phel }}\right)^{2} / 2 \sigma^{2}\right)}
$$

where $\bar{N}_{\text {phel }}$ now corresponds also to the number of photoelectrons at the maximum of the Gaussian.

The photoelectrons are accelerated by a potential difference $U$ and deposit in the silicon an average energy (if we ignore backscattering)

$$
\bar{E}=\left(E-E_{\text {dead }}\right) \sum_{m} m P_{m}\left(\bar{N}_{\text {phel }}\right)=e\left(U-U_{\text {dead }}\right) \bar{N}_{\text {phel }}
$$

where $E=e U$ is the photoelectron energy at the anode and $E_{\text {dead }}=e U_{\text {dead }}$ represents a small energy loss due to the few nanometers thick silicon dead layer $d$. The average number of generated electron-hole pairs will then be

$$
n_{e l-h}=\frac{e\left(U-U_{\text {dead }}\right)}{\varepsilon} \bar{N}_{\text {phel }}
$$

and its fluctuation (intrinsic noise)

$$
\begin{aligned}
\sigma_{\text {int }} & =\sqrt{F \frac{\left(E-E_{\text {dead }}\right)}{\varepsilon} \sum_{m} m P_{m}\left(\bar{N}_{\text {phel }}\right)} \\
& =\sqrt{F \frac{e\left(U-U_{\text {dead }}\right)}{\varepsilon} \bar{N}_{\text {phel }}}
\end{aligned}
$$

where $F \sim 0.1$ is the Fano factor (see [2]), $\varepsilon$ is defined in Section I, and $\sigma_{\text {int }}$ is expressed in $n_{e l-h}$ units.

In turn, these electron-hole pairs give rise to the electrical signals measured by a charge preamplifier [2]. Noise added to the measurement from the statistical fluctuation in energy loss of the photoelectron in the silicon and from the electronic noise modify relation $(4 a)$ to

$$
\sigma=\sqrt{\left(\bar{N}_{\text {phel }}\right)+\left[\frac{\varepsilon F}{e\left(U-U_{\text {dead }}\right)} \bar{N}_{\text {phel }}\right]+\left[\frac{\sigma_{\text {el }}}{e\left(U-U_{\text {dead }}\right)}\right]^{2}}
$$

where $\sigma_{\mathrm{el}}$ is the r.m.s. electronic noise of the preamplifier, expressed in electronvolts-equivalent, and $\sigma$ is expressed in photoelectron numbers [note the different units in (6b)]. Typical values for cross-focused HPMT's are: $U=13 \mathrm{kV}, U_{\text {dead }}=1.5$ $\mathrm{kV}, \sigma_{\mathrm{el}}=0.8 \mathrm{keV}$, therefore

$$
\sigma=\sqrt{\left(1+2.8 \cdot 10^{-5}\right) \bar{N}_{\mathrm{phel}}+0.07^{2}} \approx \sqrt{\bar{N}_{\mathrm{phel}}}
$$

3 is dominated by the photoelectron emission statistics.

The situation is quite different for Photo Multiplier Tubes (PMT's). Their standard deviation is equal to $\sigma=\sqrt{1+\sigma_{\text {ser }}^{2}} \sqrt{\bar{N}_{\text {phel }}}$, where $\sigma_{\text {ser }}$ ranges between 0.4 and 0.8 and means the single electron response standard deviation [14]. The latter stems from two major processes: one is low statistics in the electron multiplication on the first dynode and the other is due to the gain fluctuations in the dynode multiplication chain. ${ }^{4}$

Relations (4) and (5) are valid without backscattering. To take backscattering into account, we must consider the probability distributions $B_{1}(q), \ldots, B_{m}(q)$, that $1, \ldots, m$ electrons are backscattered from silicon at any angle leaving in the medium the fractional energy $q=1-E_{b} / E_{0}$. These are given by iterative self-convolutions starting with $B_{1}(q)$ (Fig. 2) [2]

$$
B_{m}(q)=\int_{0}^{q_{m-1}} B_{m-1}\left(q^{\prime}\right) B_{1}\left(q-q^{\prime}\right) d q^{\prime}
$$

where $\int B_{m}(q) d q=1$ and is justified by the fact that the probability distribution $B_{1}(q)$ for each electron is independent from the total number of incident photoelectrons. With these definitions, the measured number of photoelectrons becomes

$$
\bar{N}_{\text {meas }}=\sum_{m} P_{m}\left(\bar{N}_{\text {phel }}\right) \int_{0}^{q_{m}} q B_{m}(q) d q
$$

and its spectrum [see (I.1)] is shown in Fig. 3.

As shown in Appendix I, relation (9) can be solved and we obtain

$$
\bar{N}_{\text {meas }}=\bar{q}_{1} \bar{N}_{\text {phel }}
$$

with

$$
\vec{q}_{1}=\int_{0}^{q_{1}} q B_{1}(q) d q
$$

The average number of measured photoelectrons can therefore be calculated by simply knowing the distribution $B_{1}(q)$ and solving the integral in relation (10a). If we assume a backscattering distribution $B_{1}(q)$, similar to the one shown in [8, Fig. 5] ${ }^{5}$ (see Fig. 2) and defined in Appendix III, we obtain

$$
\left(\overline{q_{1}}\right)=1-\mu+\frac{\left(\frac{3}{4}-q_{\max }\right)}{\left(2-3 q_{\max }\right)} \mu
$$

${ }^{3}$ It is worth noting that the single electron peaks in the spectra will be described by Gaussian distributions with variances equal to $\sigma_{m}^{2}=\sigma_{\mathrm{cl}}^{2}+\sigma_{\text {int }}^{2}=$ $(0.8 \mathrm{keV})^{2}+(0.07 \cdot \mathrm{mkeV})^{2}$. Therefore, single peak separation, given by $e U$, stays constant, but peak visibility degrades with $m$ [2].

4The gain of PMT's varies with the potential difference $U_{d}$ between their $n$ dynodes, for instance, $G_{p m t} \sim\left(U_{d}\right)^{n}$. In contrast, HPT's feature linear gain, proportional to their gap voltage $G_{h p t} \sim e U[2]$.

${ }^{5}$ Note that the abscissa of [8, Fig. 5] means $E_{b} / E_{0}$, whereas in our notation it means $q=1-E_{b} / E_{0}$. 
which leads to

$$
\bar{N}_{\text {meas }}=\left(\bar{q}_{1}\right) \bar{N}_{\text {phel }}=0.88 \bar{N}_{\text {phel }}
$$

if we take $q_{\max }=\left(1-E_{b} / E_{0}\right)_{\max }=0.3$ as already previously mentioned.

A similar reasoning (see Appendix II) leads us to the value of the standard deviation for the distributions (4) and (5) (see for example, a total absorption peak obtained with an HPMT employed in a gamma detection mode)

$$
\sigma^{2}=\bar{q}_{1}^{2} \bar{N}_{\text {phel }}
$$

$\sigma^{2}$ takes now backscattering into account, via

$$
\overline{q_{1}^{2}}=\int_{0}^{q_{1}} q^{2} B_{1}(q) d q
$$

which is the variance of the $B_{1}(q)$ distribution.

For the backscattering distribution, (14) becomes

$$
\overline{q_{1}^{2}}=1-\mu+\frac{\left(\frac{2}{5}-\frac{1}{2} q_{\max }\right)}{\left(2-3 q_{\max }\right)} \mu .
$$

The energy resolution of a Gaussian distribution amounts to

$$
\left(\frac{\text { FWHM }}{\bar{N}_{\text {meas }}}\right)=2.36 \frac{\sigma}{\bar{N}_{\text {meas }}} .
$$

Replacing $\sigma / \bar{N}_{\text {meas }}$, according to (10) and (13), we obtain from (16)

$$
\left(\frac{\mathrm{FWHM}}{\bar{N}_{\text {meas }}}\right)=\frac{2.36}{\sqrt{\bar{N}_{\text {meas }}}} \sqrt{\frac{\overline{q_{1}^{2}}}{\overline{q_{1}}}}=\frac{2.36}{\sqrt{\bar{N}_{\text {phel }}}} \frac{\sqrt{\overline{q_{1}^{2}}}}{\overline{q_{1}}}
$$

where $2.36 / \sqrt{\bar{N}_{\text {phel }}}$ is the energy resolution without taking backscattering into account [see (4a)]. Therefore, by inserting (11) and (15) in (17), we obtain

$$
\begin{aligned}
\left(\frac{\text { FWHM }}{\bar{N}_{\text {meas }}}\right) & =\frac{2.36}{\sqrt{\bar{N}_{\text {phel }}}} \frac{\sqrt{1-\mu+\frac{\left(\frac{2}{5}-\frac{1}{2} q_{\max }\right)}{\left(2-3 q_{\max }\right)} \mu}}{\left[1-\mu+\frac{\left(\frac{3}{4}-q_{\max }\right)}{\left(2-3 q_{\max }\right)} \mu\right]} \\
& =1.043 \frac{2.36}{\sqrt{\bar{N}_{\text {phel }}}}
\end{aligned}
$$

for the backscattering distribution with $q_{\max }=0.3$ and $\mu=$ 0.20 . Fig. 4 shows the calculated spectra for $\bar{N}_{\text {phel }}=100$ with and without backscattering. Notice that a slight indication of photoelectron peaks on top of the no-backscattering spectrum is still visible, in spite of the high average number of photoelectrons and of the intrinsic noise $\left[\sigma_{\text {int }} \sim 2.7 \mathrm{keV}\right.$, see (6b)]. ${ }^{6}$

In the case of large anodes, as in (MAP)-tubes or in (ISPA)tubes when we use the rear contact analogue signals [15], (17) can be generalized by including the electronic- and intrinsic${ }^{6}$ For

${ }^{6}$ For examples of gamma spectra recorded with HPT's see [1], [10]-[12], and terms of (7) and calculating the correct values for $\sigma_{\mathrm{el}}$ and $U$ potentials. However, backscattering here will play a different role, as many of the backscattered photoelectrons can return and be re-absorbed by the anode, each time crossing the silicon dead layer.

\section{CONCLUSION}

From (10) and (12) we learn that the number $\bar{N}_{\text {meas }}$ of photoelectrons absorbed in the silicon anode of a cross-focused HPMT amounts to $88 \%$ of the number $\bar{N}_{\text {phel }}$ of photoelectrons emitted from the photocathode. Relations (17) and (18) indicate a broadening of photoelectron- or total absorption peaks by a factor 1.043 due to backscattering. This is less than for PMT's. For example, according to the Thorn EMI Catalogue [14], the broadening of total gamma absorption for photomultipliers amounts to an average factor of 1.18 (see [14, Table 5.4, p. 12]). This factor is due to the secondary electron emission statistics associated with a small secondary electron yield at the first dynode and to gain fluctuations on the dynode chain. Finally, this paper provides a simple formalism to calculate backscattering effects in different types of HPT's.

\section{APPENDIX I}

Calculation of the Measured Average Number of Photoelectrons With BacksCATtering PROCESS

According to (8)

$$
B_{m}(q)=\int_{0}^{q_{m-1}} B_{m-1}\left(q^{\prime}\right) B_{1}\left(q-q^{\prime}\right) d q^{\prime}
$$

where $\int B_{m}(q) d q=1$ and $q_{m}=m$ for any $m$. To calculate these distributions, we need to know $B_{1}(q)$, from measurements or simulations (Fig. 2). The resulting spectrum (Fig. 3) is their weighted summation ${ }^{7}$

$$
S\left(\bar{N}_{\text {phel }}, q\right)=\sum_{m} P_{m}\left(\bar{N}_{\text {phel }}\right) B_{m}(q) .
$$

It can be verified that

$$
\int_{0}^{q_{m}} S\left(\bar{N}_{\text {phel }}, q\right) d q=1
$$

and

$$
\int_{0}^{q_{m}} S\left(\bar{N}_{\mathrm{phel}}, q\right) q d q=\sum_{m} P_{m}\left(\bar{N}_{\mathrm{phel}}\right) \bar{q}_{m}=\bar{N}_{\text {meas }}
$$

where we replaced

$$
\int_{0}^{q_{m}} q B_{m}(q) d q=\bar{q}_{m}
$$

By inserting (8) in (I.4) and interchanging the order of the integrals, we get

$$
\bar{q}_{m}=\bar{q}_{1}+\bar{q}_{m-1}=m \bar{q}_{1} \text { where } \bar{q}_{1}=\int_{0}^{q_{1}} q B_{1}(q) d q .
$$

\footnotetext{
${ }^{7}$ For simplicity, we shall not consider in the formulae the preamplifier elec-
} tronic noise, but we will convolute it properly in the shown figures. 
Therefore, by inserting (I.5) in (I.3) and remembering (6), we obtain the final relation

$$
\bar{N}_{\text {meas }}=\sum_{m} \bar{q}_{m} P_{m}\left(\bar{N}_{\text {phel }}\right)=\bar{q}_{1} \bar{N}_{\text {phel }} .
$$

\section{APPENDIX II}

\section{Calculation of the Variance Broadening in Poissonian} PROCESSES IN PRESENCE OF ELECTRON BACKSCATTERING

Without backscattering $\sigma^{2}$ is written as

$$
\sigma^{2}=\sum_{m}\left(m-\bar{N}_{\text {phel }}\right)^{2} P_{m}\left(\bar{N}_{\text {phel }}\right)=\bar{N}_{\text {phel }}
$$

where $\sigma^{2}$ is the variance of distribution (4). With backscattering included (II.1) becomes

$$
\sigma^{2}=\sum_{m} \int_{0}^{q_{m}}\left(q-\bar{N}_{\text {meas }}\right)^{2} B_{m}(q) P_{m}\left(\bar{N}_{\text {phel }}\right) d q .
$$

Using (10) and performing wherever possible integrals and sums, we obtain

$$
\sigma^{2}=-2\left(\bar{q}_{1} \bar{N}_{\text {phel }}\right)^{2}+\sum_{m} \bar{q}_{m}^{2} P_{m}\left(\bar{N}_{\text {phel }}\right)
$$

where we have replaced

$$
\int_{0}^{q_{m}} q^{2} B_{m}(q) d q=\overline{q_{m}^{2}}
$$

Again, we insert relation (8) in (II.4), exchange the order of the integrals, and perform the integration to obtain

$$
\overline{q_{m}^{2}}=\overline{q_{1}^{2}}+2 \bar{q}_{1} \bar{q}_{m-1}+\overline{q_{m-1}^{2}}=\overline{q_{1}^{2}}+2(m-1) \bar{q}_{1}^{2}+\overline{q_{m-1}^{2}}
$$

as $\bar{q}_{m-1}=(m-1) \bar{q}_{1}$ and $\overline{q_{1}^{2}} \neq \bar{q}_{1}^{2}$.

Relation (II.5) is again a recursive equation, which can be solved, yielding

$$
\overline{q_{m}^{2}}=m \overline{q_{1}^{2}}+m(m-1) \bar{q}_{1}^{2} .
$$

Inserting (II.6) in (II.3) and performing the sums, we finally obtain (13)

$$
\sigma^{2}=\overline{q_{1}^{2}} \bar{N}_{\text {phel }}
$$

since $\sum_{m} m P_{m}\left(\bar{N}_{\text {phel }}\right)=\bar{N}_{\text {phel }}$ and $\sum_{m} m(m-$ 1) $P_{m}\left(\bar{N}_{\text {phel }}\right)=\bar{N}_{\text {phel }}^{2}$.

\section{APPENDIX III}

To roughly fit the $\mathrm{Al}$ distribution indicated in [8, Fig. 5], a parabolic-like distribution $B_{1}(q)$ is defined as

$$
B_{1}(q)=\left\{\begin{array}{l}
\frac{3 \mu}{\left(1-1.5 q_{\max }\right)}\left(0.5-q_{\max }+q_{\max } q-0.5 q^{2}\right) \\
\text { for } 0 \leq q<q_{1} \\
(1-\mu) \delta\left(q-q_{1}\right) \\
\text { for } q=q_{1}=1
\end{array}\right.
$$

and it is shown in Fig. 2 (with electronic noise added). Here $\delta\left(q-q_{1}\right)$ is the delta function and formally has a meaning only when used in an integral. It can be verified that

$$
\begin{aligned}
\int_{0}^{q_{1}} & B_{1}(q) d q \\
= & \int_{0}^{q_{1}} \frac{3 \mu}{\left(1-1.5 q_{\max }\right)}\left(0.5-q_{\max }+q_{\max } q-0.5 q^{2}\right) d q \\
& +\int_{0}^{q_{1}}(1-\mu) \delta\left(q-q_{1}\right) d q=1 .
\end{aligned}
$$

\section{ACKNOWLEDGMENT}

The authors would like to acknowledge the fruitful collaboration and the interesting discussions with our colleagues, F. De Notaristefani, D. Puertolas, and E. Rosso.

\section{REFERENCES}

[1] C. D’Ambrosio, T. Gys,, E. Heijne, H. Leutz, D. Piedigrossi, D. Puertolas, and E. Rosso, "The ISPA-tube and the HPMT, two examples of a new class of photodetectors: The hybrid photo detectors," Nucl. Phys. $B$, vol. 61B, p. 638, 1998.

[2] C. D'Ambrosio, T. Gys, H. Leutz, D. Piedigrossi, D. Puertolas, and S. Tailhardat, "Photon counting with a hybrid photomultiplier tube (HPMT)," Nucl. Instrum. Methods, vol. A338, p. 389, 1994.

[3] A. Braem, E. Chesi, W. Dulinski, F. Filthaut, A. Go, C. Joram, J. Seguinot, P. Weilhammer, H. Wenzel, P. Wicht, and T. Ypsilantis, "Highly segmented large-area hybrid photodiodes with Bialkali photocathodes and enclosed VLSI readout electronics," Nucl. Instrum. Methods, vol. A442, p. 128, 2000.

[4] C. D'Ambrosio, T. Gys, H. Leutz, D. Piedigrossi, D. Puertolas, and S. Tailhardat, "Position sensitive photon counting with an ISPA-tube," IEEE Trans. Nucl. Science, vol. 42, p. 130, 1995.

[5] H. E. Bishop, "Electron scattering in thick targets," Brit.. J. Appl. Phys., vol. 18, p. 703, 1967.

[6] H. Drescher, L. Reimer, and H. Seidel, "üäRckstreukoeffizient und Sekundrelektronen-Ausbeute von 10-100 keV-Elektronen und Beziehungen zur Raster-Elektronen-mikroskopie," Z. Angew. Phys., vol. 29, p. 331, 1970.

[7] H. Seidel, "PhD—Thesis," Ph.D. dissertation, Univ. Münster, Germany, 1973

[8] E. H. Darlington, "Backscattering of 10-100 keV electrons from thick targets," J. Phys. D: Appl. Phys., vol. 8, p. 85, 1975.

[9] H. O. Funsten, D. M. Suszcynsky, S. M. Ritzau, and R. Korde, "Response of $100 \%$ internal quantum efficiency silicon photodiodes to 200 eV-40 keV electrons," IEEE Trans. Nucl. Sci., vol. 44, p. 2561, 1997.

[10] C. D'Ambrosio, C. Ercoli, S. Jaaskelainen, G. Lecoeur, H. Leutz, R. Loos, D. Piedigrossi, D. Puertolas, E. Rosso, and R. Schomaker, "A HPMT based set-up to characterize scintillating crystals," Nucl. Instrum. Methods, vol. A434, p. 387, 1999.

[11] C. D'Ambrosio, F. De Notaristefani, H. Leutz, D. Puertolas, and E. Rosso, "Improved light transitions from scintillators to new photocathode windows," Nucl. Instrum. Methods, vol. A431, p. 455, 1999.

[12] — , "X-ray detection with a scintillating YAP-window hybrid photomultiplier tube," IEEE Trans. Nucl. Sci., vol. 1, p. 6, 2000.

[13] T. Tabarelli de Fatis, "Light spectra sum rule," Nucl. Instrum. Methods, vol. A385, p. 366, 1997.

[14] PMT's and Accessories, Thorn EMI Electron Tubes Catalogue, p. 12, 1993.

[15] D. Puertolas, D. Piedigrossi, R. Pani, H. Leutz, T. Gys, F. De Notaristefani, and C. D'Ambrosio, "An ISPA-camera for gamma rays," IEEE Trans. Nucl. Sci., vol. 42, p. 2221, 1995. 